\title{
Correlation of hybrid beamforming arrays in the context of OTA testing
}

\author{
Pekka Kyösti
}

\begin{abstract}
Hybrid beamforming antenna arrays are a prominent component in communications at $\mathbf{m m}$-wave frequencies. Multiple analogue beamforming arrays will be used for spatial multiplexing of signals also in hand held devices. In this work we derive the correlation coefficient for signals received by two ideal arrays in simple power angular distribution schemes. The purpose is to develop tools for evaluating over-the-air setups for testing of hybrid beamforming devices. We show a few exemplary correlation error evaluations that compare near field multi-probe OTA setups with a perfect target channel model. Results indicate that the correlation error increases with decreasing range length and channel angular spread. Moreover, larger inter-array spacing and sub-array sizes increase the correlation error.
\end{abstract}

Index Terms-Spatial correlation; antenna arrays; near field; testing.

\section{INTRODUCTION}

The transmission loss is high at millimetre wave (mm-wave) frequency bands. More specifically, the free space path loss increases proportional to the frequency squared [1]. Antenna arrays can and must be used to compensate the severe transmission losses by array gains [2]. Especially at mm-wave, the antenna arrays are expected to operate in the hybrid beamforming mode. Each antenna element may not be supported by separate radio frequency (RF) chains for practical implementation reasons, mainly related to the power consumption [3]. Instead, the arrays are connected to a base band unit by only a small number of RF chains. The antenna elements are divided to sub-arrays, where elements are combined to a single RF port by an analogue weighting matrix. The matrix enables composing a predefined set of fixed antenna beams. Thus each RF port, feeding a number of antenna elements (sub-array), may compose a number of predefined beam shapes. 3GPP standardization directions on $5 \mathrm{G}$ New Radio beamforming are described in [4].

The spatial correlation has been a useful measure to characterize radio channel. Clarke derived the spatial auto-covariance function of electric field in [5]. Often the spatial correlation has been considered as the correlation of signals received by antenna elements. Antennas must be assumed isotropic if we define literally the spatial correlation, not the antenna correlation. Correlation of signals received and integrated by antenna arrays has not been widely investigated. In the mentioned hybrid beamforming schemes the correlation of

This work has been partly done in 6G Flagship programme funded by Academy of Finland (grant no. 318927).

Pekka Kyösti is with Centre for Wireless Communications, University of Oulu, Finland (email: pekka.kyosti@oulu.fi). P. Kyösti is also with Keysight Technologies Finland Oy (email: pekka.kyosti@keysight.com). signals received by two spatially separated, independently analogue beamforming antenna arrays becomes interesting.

In this work we develop the spatial correlation function for two ideal arrays and discretized far field angular power distributions. We determine the corresponding correlation function also for the case of near field sources. The intention is to determine tools to evaluate the impact of a short range length [6] in the over the air (OTA) testing of radio devices [7]. The near field sources are probe antennas and the far field sources represent a target channel model in this context. Channel models and OTA methodologies for 3GPP New Radio radiated testing are described in the draft report [8].

The polarization dimension is omitted in this work. Most often signals transmitted with two orthogonal polarizations are separable by a receiver with orthogonally polarized antennas. The mutual coupling between antennas is ignored in this work.

\section{SPATIAL CORRELATION OF ARRAYS}

The spatial correlation between beamforming sub-arrays may be important in the (potentially rare) cases when: 1) more than two multiplexed signal streams are communicated and the polarization domain is not capable to separate the streams and 2) beams of sub-arrays are pointing to the same direction, i.e., same weight vectors are used for different sub-arrays. In [9] the spatial correlation coefficient for two isotropic elements in arbitrary locations was defined based on literature in the ideal case and derived assuming limited range length in a practical case. Now our intention is to derive similar formulas considering simple beamforming antenna arrays with variable inter-array spacing. The starting point for our analysis will be the array factor. A reverse relationship between array factors and correlation matrices is presented in [10], where the former is determined based on the latter.

For mainly notational simplicity, we consider linear horizontal arrays instead of planar arrays in the following analysis. However, we expect that the observations done on the impact of limited range length are applicable also with more complex array structures.

The system function for a beamforming array of $U$ elements can be written as (neglecting noise)

$$
y=\mathbf{B}_{1 \times U} \mathbf{H}_{U \times Q} \mathbf{X}_{Q \times 1},
$$

where $y$ is the combined signal, the weight vector $\mathbf{B}=$ $\left\{\gamma_{u}\right\}_{u=1 \ldots U}, \mathbf{H}$ is the transfer matrix from $Q$ sources to $U$ antenna elements, and $\mathbf{X}$ is the signal vector radiated by $Q$ sources. Furthermore, the array has element locations specified 
by position vectors $\bar{r}_{u}$. A perfect plane wave from direction of the $q$ th source is denoted by the wave vector $\bar{\beta}_{q}$. Now the array factor [11] for the direction $\bar{\beta}_{q}$ is

$$
A\left(\bar{\beta}_{q}\right)=\sum_{u=1}^{U} I_{u} e^{j\left(\bar{\beta}_{q} \cdot \bar{r}_{u}+\gamma_{u}\right)},
$$

where $I_{u}$ is the current induced to the $u$ th element, $\cdot$ denotes the dot product operation, and $\gamma_{u}$ is the phase weight of $u$ th element.

\section{A. Inter-array correlation in ideal case}

Assume an antenna system composed of two sub-arrays that are parallel and identically oriented uniform linear arrays (ULAs) $\mathrm{U}$ and $\mathrm{V}$ as illustrated in Fig. 1. They have the interarray spacing $D$ and inter-element spacing $d$. The number of ideal isotropic elements in sub-arrays is $U$ and $V$, respectively. The polarization is not in the focus of this analysis, thus we may assume all antennas are vertically polarized ideal elements with unity gain. Both sub-arrays perform beamforming independently. The signals received by elements of array $\mathrm{U}$ are combined with weights $\gamma_{u}$ and of array $\mathrm{V}$ with $\alpha_{v}$.

The complex array factors of $\mathrm{V}$ and $\mathrm{U}$ to direction $\theta_{q}$ are

$$
\left\{\begin{array}{l}
A_{\mathrm{V}}\left(\theta_{q}\right)=\sum_{v=1}^{V} e^{j\left(-\beta v d \sin \theta_{q}+\alpha_{v}\right)} \\
A_{\mathrm{U}}\left(\theta_{q}\right)=\sum_{u=1}^{U} e^{j\left(-\beta(u d+D) \sin \theta_{q}+\gamma_{u}\right)}
\end{array},\right.
$$

where $\beta=2 \pi$ /wavelength is the wave number. Assume $Q$ discrete sources (probes) radiating independent (fading) signals with directions and mean powers $\theta_{q}$ and $a_{q}^{2}, q=1, \ldots, Q$, respectively. The covariance of combined signals $C_{\mathrm{UV}}=$ $E\left[y_{\mathrm{U}} y_{\mathrm{V}}^{H}\right]$ can be developed to form

$$
\begin{aligned}
C_{\mathrm{UV}} & =\sum_{q=1}^{Q}\left(a_{q} A_{\mathrm{U}}\left(\theta_{q}\right)\right)\left(a_{q} A_{\mathrm{V}}\left(\theta_{q}\right)\right)^{*} \\
& =\sum_{q=1}^{Q}\left(a_{q}^{2} \sum_{u=1}^{U} \sum_{v=1}^{V} e^{j\left(\beta(v d-u d-D) \sin \theta_{q}+\gamma_{u}-\alpha_{v}\right)}\right),
\end{aligned}
$$

where ()$^{*}$ denotes the complex conjugate operation.

It is assumed that the sub-arrays perform analogue beamforming with a pre-defined code book, i.e., with a limited set of weight vectors. Further, it is assumed that both subarrays are perfectly calibrated. In the following analysis of this section, we consider only the case $\gamma_{u}=\alpha_{v}=0$, i.e., the beams are pointing to the broad side direction of arrays, and drop out the weight terms. This limitation is taken, since calculating correlation coefficients with different beamforming weights would expand the number of considered alternatives very high. ${ }^{1}$

The auto-covariance of sub-array $\mathrm{U}$ is

$$
C_{\mathrm{UU}}=\sum_{q=1}^{Q}\left(a_{q}^{2} \sum_{u=1}^{U} \sum_{u^{\prime}=1}^{U} e^{j\left(\beta\left(u^{\prime}-u\right) d \sin \theta_{q}+\gamma_{u}-\gamma_{u^{\prime}}\right)}\right)
$$

${ }^{1}$ We expect that if arrays $\mathrm{U}$ and $\mathrm{V}$ applied different weight vectors, i.e. $\gamma_{u} \neq \alpha_{u}$, the arrays were further de-correlated and the impact of possible limitations of the test setup became less significant anyhow. and the auto-covariance $C_{\mathrm{VV}}$ is defined correspondingly. If $U=V$ then $C_{\mathrm{VV}}=C_{\mathrm{UU}}$.

Finally the cross-correlation coefficient for sub-arrays $U$ and $\mathrm{V}$ in the ideal far field condition, when $\gamma_{u}=\alpha_{v}=0 \forall u \in$ $\mathcal{U}, v \in \mathcal{V}$, is

$$
\begin{aligned}
& \rho_{\mathrm{UV}}=\frac{C_{\mathrm{UV}}}{\sqrt{C_{\mathrm{UU}} C_{\mathrm{VV}}}}= \\
& \frac{\sum_{q \in \mathcal{Q}, u \in \mathcal{U}, v \in \mathcal{V}}\left(a_{q}^{2} e^{j \beta(v d-u d-D) \sin \theta_{q}}\right)}{\sqrt{\sum_{q, q^{\prime} \in \mathcal{Q}, u, u^{\prime} \in \mathcal{U}, v, v^{\prime} \in \mathcal{V}}\left(a_{q}^{2} a_{q^{\prime}}^{2} e^{j \beta\left(u^{\prime}-u+v^{\prime}-v\right) d \sin \theta_{q}}\right)}},
\end{aligned}
$$

where the index set $\mathcal{Q}=\{1, \ldots, Q\}, \mathcal{U}=\{1, \ldots, U\}$, and $\mathcal{V}=\{1, \ldots, V\}$

\section{B. Inter-array correlation in limited range length case}

The settings and assumptions are identical to the ideal case, except that now the $Q$ sources are located in a coordinate system. The configuration is illustrated in Fig. 2. The correlation is affected both by the varying angles $\theta_{u q}$ and $\theta_{v q}$ and path loss variation resulting from distances $d_{u q}$ and $d_{v q}$. The location of probe $q$ is specified by the distance $R$ and angle $\sigma_{q}$ as observed from the centre of the test zone, i.e., midpoint of the antenna system.

The transfer function from probe $q$ to element $v$ is defined simply, assuming unity antenna gains and the free space path loss [1], as

$$
h_{v q}=\frac{1}{2 \beta d_{v q}} e^{j \beta d_{v q}} .
$$

The inter-array cross-covariance of combined signals from $Q$ probes is

$$
\tilde{C}_{\mathrm{UV}}=\frac{1}{4 \beta^{2}} \sum_{q=1}^{Q}\left(a_{q}^{2} \sum_{u=1}^{U} \sum_{v=1}^{V} \frac{e^{j\left(\beta\left(d_{u q}-d_{v q}\right)+\gamma_{u}-\alpha_{v}\right)}}{d_{u q} d_{v q}}\right)
$$

and the auto-covariance is

$$
\tilde{C}_{\mathrm{UU}}=\frac{1}{4 \beta^{2}} \sum_{q=1}^{Q}\left(a_{q}^{2} \sum_{u=1}^{U} \sum_{u^{\prime}=1}^{U} \frac{e^{j\left(\beta\left(d_{u q}-d_{u^{\prime} q}\right)+\gamma_{u}-\gamma_{u^{\prime}}\right)}}{d_{u q} d_{u^{\prime} q}}\right) .
$$

The correlation between sub-arrays $\mathrm{U}$ and $\mathrm{V}$, limiting to the case of zero weights, can be developed to form

$$
\begin{aligned}
& \tilde{\rho}_{\mathrm{UV}}= \\
& \sqrt{\sum_{q \in \mathcal{Q}, u \in \mathcal{U}, v \in \mathcal{V}}\left(a_{q}^{2} \frac{e^{j \beta\left(d_{u q}-d_{v q}\right)}}{d_{u q} d_{v q}}\right)} \\
& .
\end{aligned}
$$

With definitions of Fig. 2 the distance between element $u$ and probe $q$ is

$$
d_{u q}=\sqrt{R^{2} \cos \sigma_{q}+\frac{1}{4}\left(d(2 u-U-1)+D-2 R \sin \sigma_{q}\right)^{2}}
$$




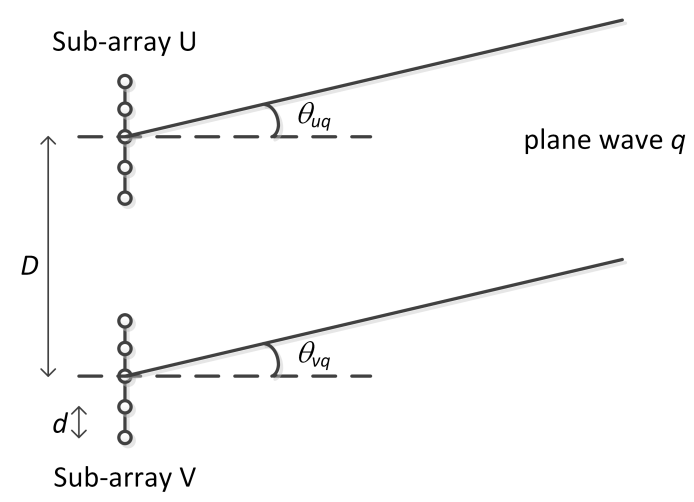

Fig. 1. Definitions for inter-array correlation in the ideal plane wave case.

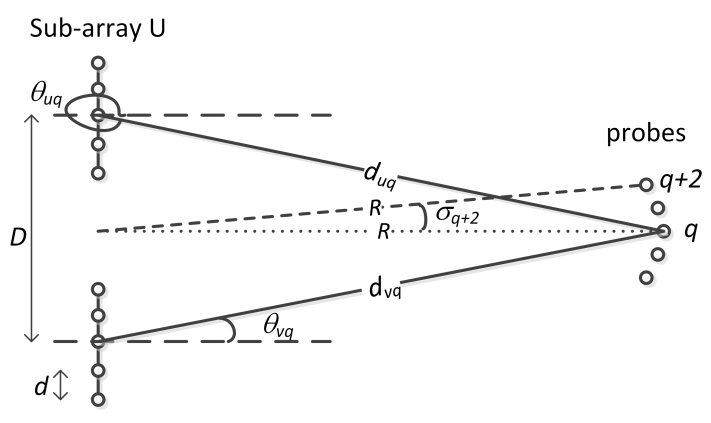

Sub-array $\mathrm{V}$

Fig. 2. Definitions for inter-array correlation in the spherical wave case.

and the distance between element $v$ and probe $q$ is

$d_{v q}=\sqrt{R^{2} \cos \sigma_{q}+\frac{1}{4}\left(d(-2 v+U+1)+D+2 R \sin \sigma_{q}\right)^{2}}$.

Alternatively, the antenna element and probe locations can be specified by coordinate points or position vectors. In this case, the distances can be determined with simple analytical geometry or vector norm calculations.

\section{RESULTS}

Spatial correlation coefficients are calculated for various inter-array spacings $D$, sub-array sizes $U(=V)$, range lengths $R$, probe sector widths $\Psi$ and offsets $\psi$ from the broad side direction. The offset parameter $\psi$ indicates how much the centre of the probe array is rotated w.r.t. the broad side of $\mathrm{U}$ and V. In the illustration of Fig. 2 the offset $\psi=0^{\circ}$.

In this analysis the angular spacing of probes is always one degree and the radiated power across sources $q \in \mathcal{Q}$ is uniform (i.e. $a_{q} \equiv 1$ ). The inter-element spacing within sub-arrays is always $d=0.5 \lambda$ and the frequency is $28 \mathrm{GHz}$. The correlation error is calculated as

$$
\rho_{\text {error }}=|\rho(D, U, R, \Psi, \psi)-\tilde{\rho}(D, U, R, \Psi, \psi)| \in[0,2] .
$$

Ranges of the parameters are specified in Table I. The correlation error is calculated as specified in eq. (13) for all
TABLE I

THE RANGE OF PARAMETERS IN

spatial correlation analysis.
\begin{tabular}{|l|c|c|c|}
\hline Parameter & symbol & min & $\max$ \\
\hline inter-array spacing & $D$ & $0 \mathrm{~m}$ & $0.2 \mathrm{~m}$ \\
sub-array size & $U / V$ & 1 & 20 \\
range length & $R$ & $0.1 \mathrm{~m}$ & $1 \mathrm{~m}$ \\
probe sector width & $\Psi$ & $1^{\circ}$ & $90^{\circ}$ \\
probe sector offset & $\psi$ & $0^{\circ}$ & $45^{\circ}$ \\
\hline
\end{tabular}

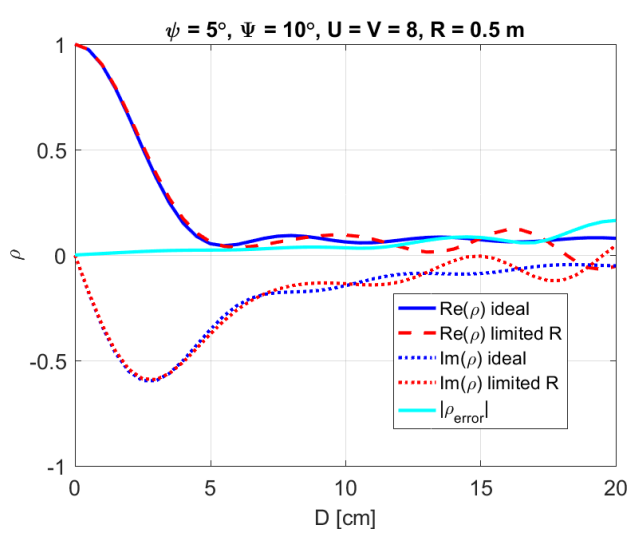

Fig. 3. Example spatial correlation curves in the ideal and practical cases as a function of inter array spacing.

combinations of the five-dimensional parameter space. The maximum error over the full parameter space is 1.998 , which results in the case of almost unity absolute correlations values (i.e. minimal magnitude error) on both $\rho$ and $\tilde{\rho}$, but almost $180^{\circ}$ phase deviation (=phase error).

The impact of varied parameters on the resulting sub-array correlation error are illustrated in Figures from 3 to 9. As five-dimensional spaces are difficult to visualize the results are shown by fixing three or four parameters and letting only two or one parameter to vary. Fig. 3 shows example correlation curves as a function of $D$ with all other four parameters fixed to values indicated in the title of the figure. Then surface plots of Fig. 4 to 9 visualize the correlation error as a function of parameter pairs like, e.g., the range length and offset angle.

The following general trends can be observed. The correlation error increases with increasing $D, U, V$, and $\psi$, and with decreasing $R$ and $\Psi$. In other words, larger inter-array spacing, larger sub-arrays and stronger power angular spectrum (PAS) offset from the broad side of arrays provides higher correlation error. Moreover, narrower PAS and evidently a shorter range length results also to higher correlation error.

\section{CONCLUSIONS}

In this work we derived spatial correlation functions for two spatially separated ULAs. This was done both for the ideal case of far field signal sources that represent a target channel model, and for the near field case where sources represent probe antennas. We showed some exemplary results of correlation error evaluations, though the main contribution are the correlation functions. 


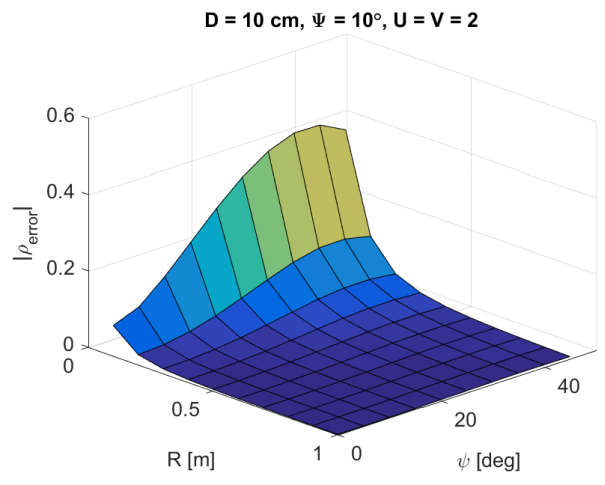

Fig. 4. Correlation error as a function of the offset angle $\psi$ and range length $R$.

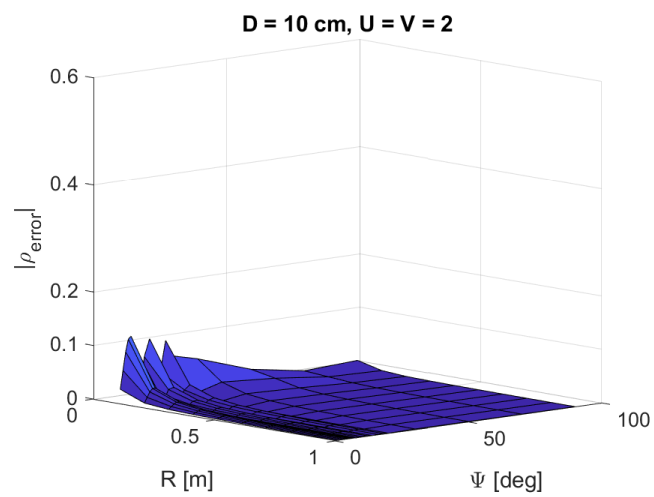

Fig. 5. Correlation error as a function of the sector width $\Psi$ and range length $R$.

At least two messages can be extracted. The first one might be evident. The spatial correlation between arrays is not identical to the spatial correlation between two spatial locations. The second is that the error of complex correlation can be high because of phase term while the absolute correlation coefficient can be close to the target value. Therefore, it is good to be careful when evaluating complex correlation errors.

As a numerical result we could mention that, when $R=$ $0.5 \mathrm{~m}$ and the sub-array spacing $D$ is limited to $10 \mathrm{~cm}$, the maximum error is 0.11 .

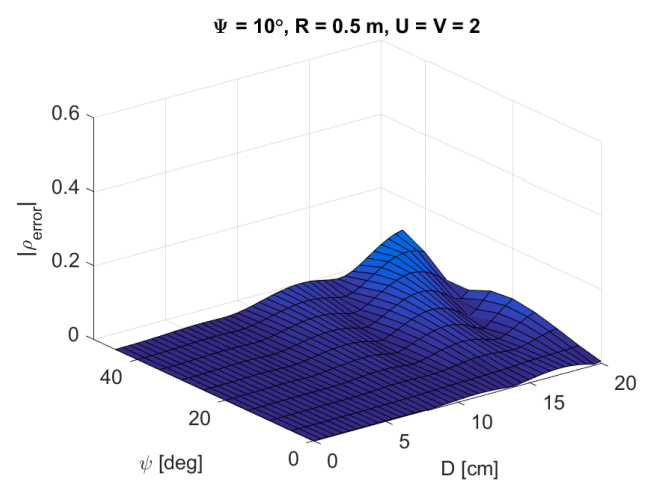

Fig. 6. Correlation error as a function of the offset angle $\psi$ and inter array spacing $D$.

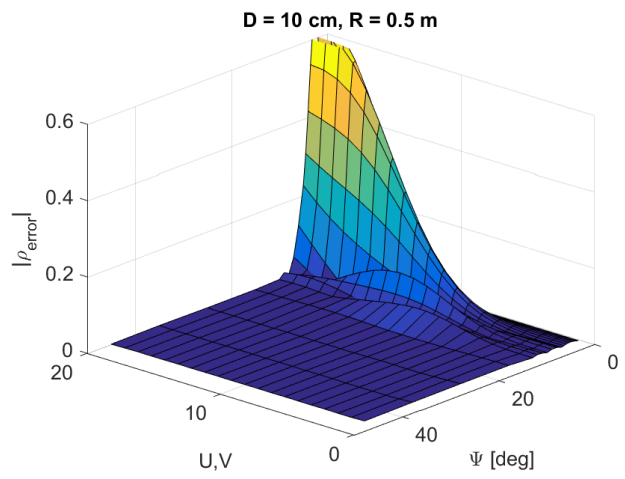

Fig. 7. Correlation error as a function of the sector width $\Psi$ and number of sub-array elements $U, V$.

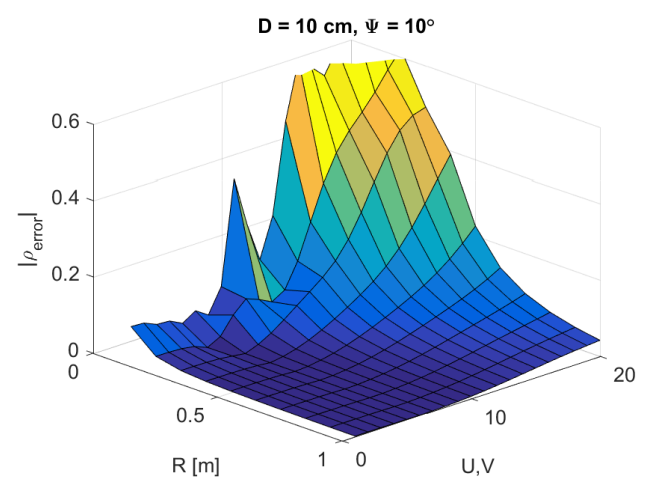

Fig. 8. Correlation error as a function of the range length $R$ and number of sub-array elements $U, V$.

\section{REFERENCES}

[1] H. T. Friis, "A note on a simple transmission formula," Proceedings of the IRE, vol. 34, no. 5, pp. 254-256, May 1946.

[2] K. Haneda, C. Gustafson, and S. Wyne, "60 ghz spatial radio transmission: Multiplexing or beamforming?" IEEE Transactions on Antennas and Propagation, vol. 61, no. 11, pp. 5735-5743, Nov 2013.

[3] S. Kutty and D. Sen, "Beamforming for millimeter wave communications: An inclusive survey," IEEE Communications Surveys Tutorials, vol. 18, no. 2, pp. 949-973, Secondquarter 2016.

[4] E. Onggosanusi, M. S. Rahman, L. Guo, Y. Kwak, H. Noh, Y. Kim, S. Faxer, M. Harrison, M. Frenne, S. Grant, R. Chen, R. Tamrakar, and a. Q. Gao, "Modular and high-resolution channel state information and

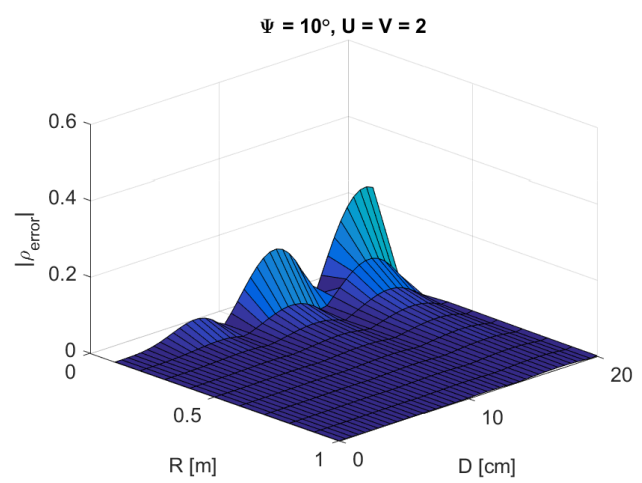

Fig. 9. Correlation error as a function of the inter array spacing $D$ and range length $R$. 
beam management for 5g new radio," IEEE Communications Magazine, vol. 56, no. 3, pp. 48-55, March 2018.

[5] R. H. Clarke, "A statistical theory of mobile-radio reception," The Bell System Technical Journal, vol. 47, no. 6, pp. 957-1000, July 1968.

[6] P. Kyösti, J. Kyröläinen, and W. Fan, "Assessing measurement distances for OTA testing of massive MIMO base station at $28 \mathrm{GHz}$," in 2017 11th European Conference on Antennas and Propagation (EUCAP), March 2017, pp. 3679-3683.

[7] P. Kyösti, L. Hentilä, W. Fan, J. Lehtomäki, and M. Latva-aho, "On radiated performance evaluation of massive MIMO devices in multiprobe anechoic chamber OTA setups," IEEE Transactions on Antennas and Propagation, vol. 66, no. 10, pp. 5485-5497, Oct 2018.

[8] TR 38.827, "Study on radiated metrics and test methodology for the verification of multi-antenna reception performance of NR User Equipment (UE); (release 16)," 3GPP, Tech. Rep. V1.0.0, December 2019.

[9] P. Kyösti and L. Hentilä, "Criteria for Physical Dimensions of MIMO OTA Multi-Probe Test Setup," in Proc. of the 6th European conference on antennas and propagation, EuCAP 2012, Prague, Czech Republic, March 2012

[10] D. Reed and A. Rodriguez-Herrera, "Array factor derived from a correlation matrix," in 2019 13th European Conference on Antennas and Propagation (EuCAP), March 2019, pp. 1-5.

[11] W. L. Stutzman and G. A. Thiele, Antenna Theory and Design, 3rd ed USA: Wiley, 2012 